\title{
Integrated micro- and nano-optical biosensor Silicon devices CMOS compatible
}

\author{
L. M. Lechuga ${ }^{*}$, B. Sepúlveda ${ }^{\mathrm{a}}$, J. Sánchez del Río $^{\mathrm{a}}$, F. Blanco ${ }^{\mathrm{b}}$, A. Calle ${ }^{\mathrm{a}}$ and C. Domínguez ${ }^{\mathrm{a}}$ \\ ${ }^{a}$ Biosensors Group. Microelectronics National Center (CNM), CSIC, Spain. Isaac Newton, 8.PTM- \\ 28760, Tres Cantos, Madrid. Spain \\ ${ }^{\mathrm{b}}$ IKERLAN S. Coop. MEMS/MST Department. E-20500 Mondragón, Guipúzcoa, Spain
}

\begin{abstract}
We show the design, fabrication and testing of micro/nanobiosensor devices based on optical waveguides in a highly sensitive interferometric configuration and by using evanescent wave detection. The devices are fabricated by standard Silicon CMOS microelectronics technology after a precise design for achieving a high sensitivity for biosensing applications. Two integrated Mach-Zehnder interferometric (MZI) devices, using two technologies, have been developed: (a) a MZI Microdevice based on ARROW waveguide (b) a MZI Nanodevice based on TIR waveguide. Direct biosensing with both sensors has been tested, after a specific receptor coupling to the surface device using nanometer scale immobilization techniques. Further integration of the microoptical sensors, the microfluidics, the photodetectors and the CMOS electronics will render in a lab-on-a-chip microsystem.
\end{abstract}

Keywords: Optical biosensor, Mach-Zehnder interferometer, Integrated optics, BIOMEMS, optical waveguides. immobilization at nanometer scale

\section{INTRODUCTION}

There is an increasing interest in systems based on micro/nanotechnologies for ultrasensitive and miniaturized biosensors. Genomics and proteomics sensing are fields where new laboratory analysis (faster, direct, more accurate, selective, of high throughput and cheaper than conventional methods) are demanded. On the other hand, new methods allowing label-free and real time measurement of simultaneous interactions (as antigen/antibody or DNA hybridization) must be developed. A highly multidisciplinary approach including microelectronics, MEMS, micro/nanotechnologies, molecular biology, nanobiotechnology and chemistry are needed for the implementation of such new analytical devices. Biosensing devices fabricated with optoelectronics micro/nanotechnologies are powerful devices which can fulfill these requirements.

Integrated optical (IO) devices are increasingly been used as transducers for optochemical sensing applications ${ }^{1}$. IO devices combine high sensitivity, mechanical stability, miniaturization and the possibility of mass-production. The used of standard Silicon microelectronics technology for the fabrication of these devices allow a high homogeneity of the waveguide material and the possibility for integration of optical, fluidics and electrical functions on one chip in order to obtain a complete microsystem.

Most of the integrated optical sensors make use of the evanescent field detection principle for sensing ${ }^{2}$. The evanescent wave (EW) techniques, as illustrated in Fig. 1, allows the direct monitoring of small changes in the optical properties and are particularly useful in the direct detection of biomolecular interaction. The direct detection method is not as sensitive as indirect ones (i.e. fluorescence, radiolabeling or enzyme amplification) but it requires, generally, no prior sample preparation and can be use in real time evaluations allowing the determination of concentration, kinetic constants and binding specificity of biomolecules. These EW sensors show a great potential for sensing biomolecular

*1aura@imm.cnm.csic.es;phone 34-918060789; fax 34-918060701; imm.cnm.csic.es/biosensores 
interactions in real-time without labeling requirements, which have made them quite useful for applications ranging from biomedical, environmental, industrial, chemical and biological warfare to genomic and proteomics fields. This technology can be an alternative to conventional analytical techniques because they can avoid expensive, complex and time-consuming procedures. In addition, the integrated optoelectronics EW sensors can be further integrated on silicon to render in a complete BIOMEMS device.

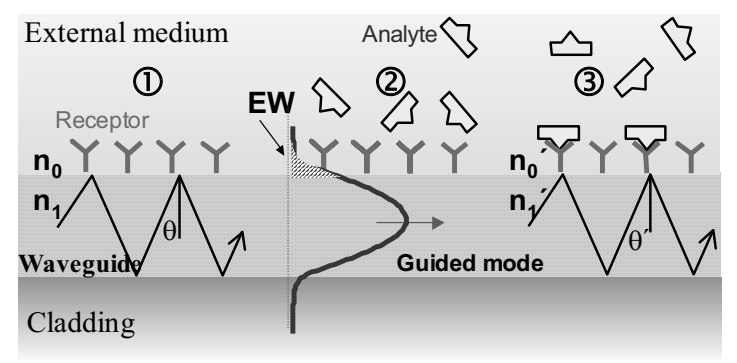

Fig. 1. Biomolecular interaction sensing by the evanescent wave detection principle in an optical waveguide sensor

For evaluation of specific interactions, the receptor is covalently attached to the sensor surface, while the complementary molecule binds to the receptor from free solution. The recognition of the complementary molecule by the receptor causes a change in the refractive index and the sensor monitors that change. After the molecular interaction, the surface can be regenerated using a suitable reagent in order to remove the bound analyte without denaturing the immobilized receptor. An extensive range of analytes can be detected using evanescent principle, from hormone disrupters, proteins, DNA, viruses to pesticides ${ }^{1}$.

There are different types of IO sensors ${ }^{3-5}$, but the interferometric arrangement is highly sensitive and is the only one that provides with an internal reference for compensation of refractive-index fluctuations and unspecific adsorption. Interferometric sensors have a broader dynamic range than most other types of sensors and show higher sensitivity as compared to other integrated scheme ${ }^{6}$. Due to the high sensitivity of the interferometer sensor the direct detection of small molecules (as for example environmental pollutants where concentrations down to $0.1 \mathrm{ng} / \mathrm{ml}$ must be detected) would be possible with this device. Detection limit is generally limited by electronic and mechanical noise, thermal drift, light source instabilities and chemical noise. But the intrinsic reference channel of the interferometric devices offers the possibility of reducing common mode effects like temperature drifts and non-specific adsorptions. Detection limit of $10^{-7}$ in refractive index (or better) can be achieved with these devices ${ }^{6,7}$ which opens the possibility of development of highly sensitive devices, for example, for in-situ pollutant detection.

The main drawback in the development and possible commercialization of the integrated MZI device is the complexity of the design, fabrication and optical adjustments. But the utilization of micro/nanotechnology for the integration on Silicon of these devices offers some advantages as better control of the light path by the use of optical waveguides, mechanical stability, higher sensitivity, miniaturization and the possibility of mass-production.

We are focus on the development of integrated optical sensors and biosensors based on micro/nanotechnologies, with high sensitivity and selectivity for application in the environment and/or the functional genomics and proteomics fields. We have fabricated two integrated Mach-Zehnder interferometric devices based on evanescent wave detection using two technologies: (a) a MZI Microdevice based on ARROW (Anti Resonant Reflecting Optical Waveguide) (b) a MZI Nanodevice based on TIR (Total Internal Reflection) waveguide. Both devices are based on symmetric channel waveguide Mach-Zehnder interferometer configuration and has been designed for having a high sensitivity towards biochemical interactions. The devices are fabricated by standard silicon technology using a CMOS compatible process. A complete microsystem must incorporate together: the micro/nanodevices, the flow cells, the modulation system, the light sources and the photodetectors with the CMOS processing electronics. Several steps are undergoing for achieving that. The flow cells are specifically designed and fabricated using a novel fabrication method $^{8}$ of 3 -D embedded microchannels using the polymer SU-8 as structural material. Several modulation schemes into the reference arm (as 
magneto-optical compensation) are under study ${ }^{9}$. Integration of sources will be achieved by connection of optical fibers or using embedded gratings.

\section{PRINCIPLE OF OPERATION OF INTERFEROMETRIC SENSORS}

\subsection{Integrated Mach-Zehnder Interferometer}

In the integrated interferometric arrangement (see Figure 2) two light beams of equal intensity are made to travel across two areas of a waveguide (one is the sensor and the other is the reference) and finally they are combined, creating an interference pattern of dark and light fringes. When a chemical or biochemical reaction takes place in the sensor area, only the light that travels through this arm will experience a change in its effective refractive index. At the sensor output, the intensity (I) of the light coming from both arms will interfere, showing a sinusoidal variation that depends on the difference of the effective refractive indexes of the sensor and reference arms $(\Delta N)$ and on the interaction length (L):

$$
I \propto[1+V \cdot \cos \Delta \Phi]
$$

Where $\mathrm{V}$ is the visibility factor and $\Delta \Phi=\left(\Phi_{r}-\Phi_{s}\right)$ is the phase shift between guided modes in the reference and sensing arms, respectively,

$$
\Delta \Phi=\frac{2 \pi}{\lambda} \cdot L \cdot \Delta N
$$

Where $\lambda$ is the wavelength. This sinusoidal variation can be directly related to the concentration of the analyte to be measured. The visibility factor gives the contrast of the interference signal (difference between the maximum and minimum intensity) and depends on the coupling factor of the divisor and on the propagation losses of the guided mode in the interferometer arms. To obtain a maximum visibility factor it is important to design a divisor or Y-junction with a coupling factor of $3 \mathrm{~dB}$ which allows that input light is equally divided in each branch of the interferometer. Moreover, propagation losses in the sensor and reference arm should be identical.

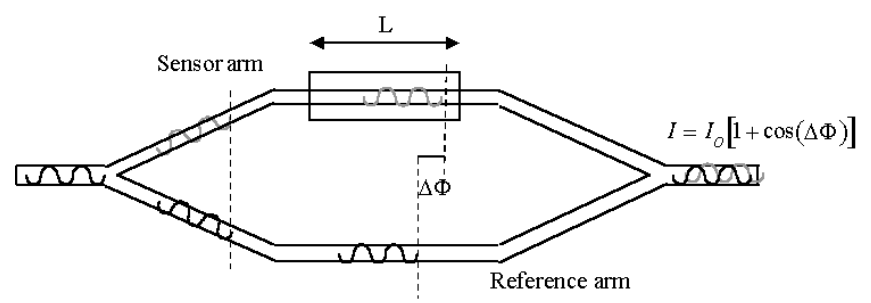

Fig. 2. Mach-Zehnder interferometer configuration

\subsection{Modeling of MZI devices for biosensing}

To obtain an efficient transducer for detecting molecular interactions, the optical waveguides of the interferomer must verify two main conditions: a high Surface Sensitivity and a Monomode behaviour ${ }^{10}$.

\subsubsection{High surface sensitivity}

A high surface sensitivity means that the sensor response for changes in the optical properties of the outer medium must be as high as possible Then, the strength and distribution of the evanescent field in the outer medium need to be maximized for giving a high response for changes in the optical properties of the surrounding medium. The biosensor to 
be developed must detect the biochemical reactions between a receptor molecule and its complementary analyte. This bio-interaction takes place on the waveguide surface and the size of the involved elements is around a few nanometers, much less than the field penetration into the outer medium (a hundred of nanometers for wavelengths in the visible). For that reason, it is very important to maximize the power value of the guided mode at the core-cover interface (the surface sensitivity). For example, to measure in a direct way a bio-interaction that involves a change in the molecular layer thickness of $0.01 \mathrm{~nm}$, the sensor should detect a change of the effective refractive index of $\Delta \mathrm{N} \approx 4 \cdot 10^{-6}$.

Surface sensitivity for processes that involve the adsorption of molecules is defined as the rate of change of the effective refractive index of the guided mode, $\mathrm{N}$, as the thickness of the homogeneous molecular adlayer, $\mathrm{d}_{\mathrm{l}}$, varies. This sensitivity is related to the squared field magnitude of the guide mode at the core-outer medium interface, considering a homogeneous adsorbed layer of refractive index $\mathrm{n}_{0}$ and thickness $\mathrm{d}_{1}$. Surface sensitivity can be expressed as a function of the power fraction in the evanescent field:

$$
S_{\text {sup }} \equiv \frac{\partial N}{\partial d_{\ell}} \approx \frac{\gamma_{o}}{N} \cdot\left[\left(\frac{n_{\ell}}{n_{o}}\right)^{2 \rho} \gamma_{o}^{2}-\left(\frac{n_{o}}{n_{\ell}}\right)^{2 \rho} \gamma_{\ell}^{2}\right] \cdot \frac{P_{o}}{P_{T}}
$$

with $\gamma_{i}=k_{o} \sqrt{N^{2}-n_{i}^{2}}(\mathrm{i}=\mathrm{o}, 1)$, where $n_{o}$ is the refractive index of the outer medium, $n_{1}$ is the refractive index of adsorbed layer, $N$ is the effective refractive index of the guided mode, $\mathrm{P}_{0} / \mathrm{P}_{\mathrm{T}}$ is the power fraction of the guided mode at the cover medium and $\rho$ is 0 for TE mode and 1 for TM mode.

The thickness and refractive indices of the waveguide layers must be designed in such a way that a large fraction of the guided mode travels through the outer medium. Using a homemade computational program based on the Non-Uniform Finite Difference Method (UN-FDM), surface sensitivity, both for TIR and ARROW structures, has been evaluated as a function of the thickness and refractive index of the core.

\subsubsection{Monomode behaviour}

Due to the evanescent sensing approach employed by the sensors, the optical waveguides must be monomode. If several modes were propagated through the structure, each of them would detect the variations in the characteristics of the outer medium and the information carried by all the modes would interfere between them. To theoretically evaluate this characteristic, we use the home-made program based on the Non-Uniform finite difference method. In channel waveguides, monomode behaviour depends on the core thickness, on the width and depth of the channel and on the difference between the core and cladding refractive indices (index contrast $\Delta n=\left(n_{c}^{2}-n_{s}^{2}\right) / n_{c}^{2}$ ).

If the difference of the core-cladding refractive index is large ( $\Delta \mathrm{n}$ higher than $10 \%)$, monomode behaviour is achieved with core thickness of hundreds of nanometers. The cladding thickness can be of only a few micrometers due to the small penetration of the evanescent field into the cladding. However, the rib depth must be around several nanometers for single-mode waveguides for a TIR waveguide with a core thickness $d_{c}=280 \mathrm{~nm}$ and index contrast $\Delta \mathrm{n}=40 \%$. Due to the nanometer dimensions of the core thickness, insertion losses for direct coupling of light with commercial optical fibers (core size between 4 and $10 \mu \mathrm{m}$, depending on the design wavelength) are high. Then, assuming a fixed wavelength, the monomode behaviour can be controlled with a proper design of the thickness and refractive indexes of the waveguide layers.

\subsubsection{MZI sensors configuration}

As a summary of the previous results, the following devices, based on two optical waveguides technologies, have been designed:

(a) a MZI Nanodevice based on TIR waveguide.

If we want to use TIR waveguides for the sensors, we must come to an agreement between single-mode behaviour, low attenuation losses for the fundamental mode and high surface sensitivity. For those reasons, the structure that has been 
finally chosen, for an operating wavelength of $0.633 \mu \mathrm{m}$, has the following structure: (i) a conducting Si wafer of 500 $\mu \mathrm{m}$ thickness, (ii) a $2 \mu \mathrm{m}$ thick thermal Silicon-Oxide layer on top with a refractive index of 1.46, (iii) a LPCVD Silicon Nitride layer of $250 \mathrm{~nm}$ thickness and a refractive index of 2.00, which is used as a guiding layer. To achieve monomode behaviour is needed to define a rib structure, with a depth of only $4 \mathrm{~nm}$, on the Silicon Nitride layer by a lithographic step. This rib structure is performed by RIE and is the most critical step in the microfabrication of the device. Finally, a Silicon-Oxide protective layer is deposited by LPCVD over the structure with a $2 \mu \mathrm{m}$ thickness and a refractive index of 1.46, which is patterning and etching by RIE to define the sensing arm of the interferometer. The final devices (within all its fabrication processes) are CMOS compatible. These devices have shown a Surface Sensitivity of $2 \cdot 10^{-4} \mathrm{~nm}-1$, close to the maximum reported up to now ${ }^{7}$. In Figure 3 the cross-section of the MZI TIR waveguide is shown.

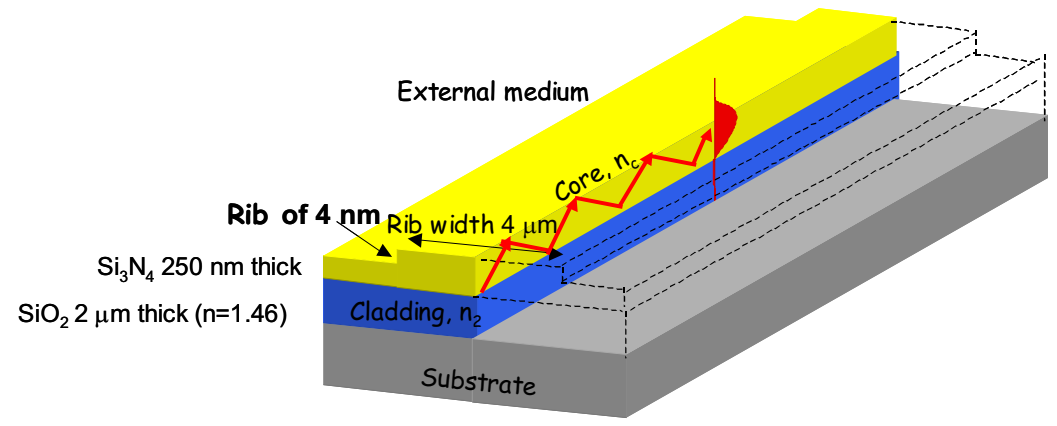

Fig. 3 Structure of the TIR-MZI device

\section{(a) MZI Microdevice based on ARROW waveguide}

In the ARROW configuration, light confinement is based on Anti-Resonant Reflections rather than Total Internal Reflection (TIR). The optical confinement of light in these waveguides is based on the total internal reflection at the aircore interface and a very high reflectivity, of $99.96 \%$, at the two interference cladding layers underneath the core ${ }^{12}$ (see Fig. 4). Each layer behaves as a quarter wave plate and, for a given wavelength, their refractive indexes and thickness have to be accurately selected. This structure is a kind of leaky waveguide (it does not support guided modes) that have an effective single-mode behaviour because higher order modes are filtered out by loss discrimination due to the low reflecting of the interference cladding. These waveguides exhibit low losses and permit larger dimensions than conventional waveguides (micrometers instead of nanometers). They have good discrimination against higher-order modes and, therefore, show virtual monomode behaviour. Moreover, they show a selective behaviour in polarisation and wavelength and a high tolerance for the selection of the refractive index and thickness of the interference layers.

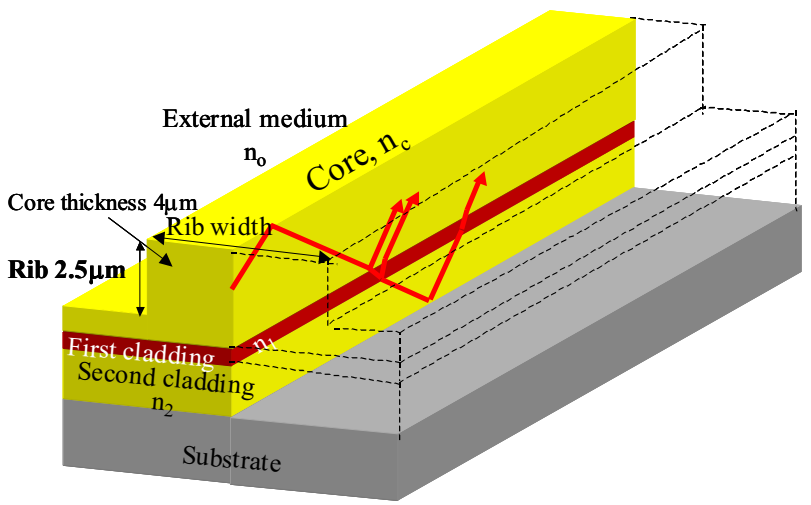

Fig.4. Cross-section of the optimized ARROW structure 
For the development of a highly sensitive integrated optical sensor based on the Mach-Zehnder interferometer configuration, we have designed an ARROW configuration which verifies the two conditions of monomode behaviour and high surface sensitivity ${ }^{13}$. This optimised waveguide consist on a rib-ARROW structure with a silicon oxide core layer $\left(\mathrm{n}_{\text {core }}=1.485\right)$ and thickness higher than $2 \mu \mathrm{m}$; a silicon oxide second cladding layer with a refractive index of 1.46 and a fixed thickness of $2 \mu \mathrm{m}$ and a silicon nitride first cladding layer, $0.12 \mu \mathrm{m}$ thick, with a refractive index of 2.00 . The waveguide is overcoated with a thin silicon nitride layer $\left(\mathrm{n}_{\mathrm{ov}}=2.00\right)$ and with a silicon oxide layer $(\mathrm{n}=1.46)$ with a thickness of $2 \mu \mathrm{m}$. The rib depth is $60 \%$ of the core thickness and the rib width should be lower than $8 \mu \mathrm{m}$ to obtain single-mode behaviour. The structure of the ARROW device can be seen in Fig. 4.

This ARROW waveguiding concept came to solve the two main limitations of the conventional TIR waveguides: the reduced dimensions for monomode behaviour (an important subject for further technological development and massproduction of the sensors) and the high insertion-losses in the optical-interconnects fiber-waveguide. With ARROW structures we can have monomode behaviour with a core thickness of the same size as the core of a single-mode optical fiber, suitable for efficient end-fire coupling. But a comparison between the evaluated surface sensitivity for both types of optical waveguides (TIR versus ARROW) as can be seen in Fig. 5, shows the higher sensitivity of the TIR device. Both designs, TIR and ARROW have advantages and disadvantages, and depending on the specific application one or another can be employed.

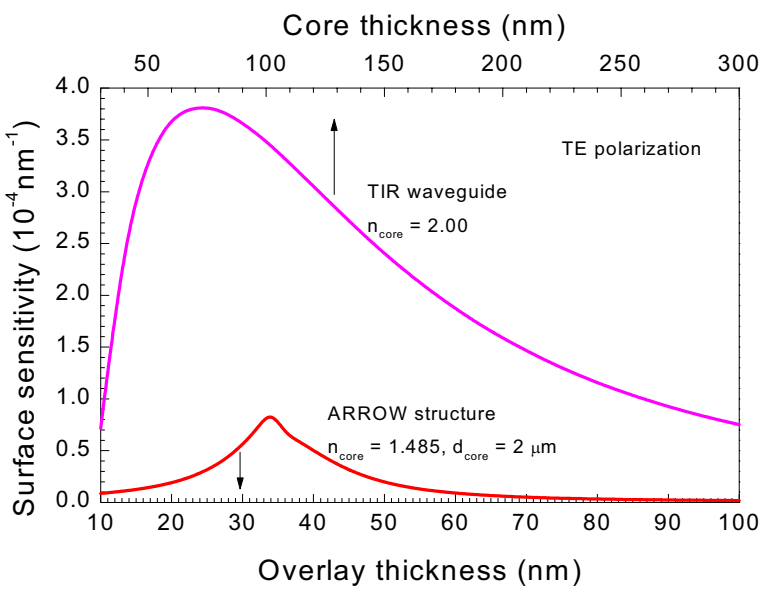

Fig. 5. Comparison of sensitivities between TIR and ARROW optical waveguides for biosensing.

\section{FABRICATION OF THE SENSOR}

The devices are fabricated in our Clean Room facilities using a Silicon CMOS compatible process. For both devices, the waveguide substrate is Silicon $\left(\mathrm{n}_{\mathrm{s}}=3.85-\mathrm{j} 0.019\right)$. Cladding and core layers $\left(\mathrm{SiO}_{2}, \mathrm{Si}_{3} \mathrm{~N}_{4}, \mathrm{SiOx}\right)$ are grown by PECVD or LPCVD. To obtain monomode lateral confinement of light, a rib structure is defined on the core layer by Reactive Ion Etching (RIE). Several devices were designed with different widths, ranging from 4 to $7 \mu$ m, to experimentally analyse its influence in the guiding properties of the structure. The sensing process (change in the optical properties of the outer medium) will take place during a certain distance, L, in the sensor arm of the interferometer. For that reason, the rest of the MZI is protected from the environment with a covering layer and the sensing window of length $\mathrm{L}$ is opened in one of the interferometer branches. The protective layer is a silicon oxide layer with a refractive index of 1.46 deposited by PECVD at $300{ }^{\circ} \mathrm{C}$. The thickness of this layer is $2 \mu \mathrm{m}$, enough to isolate the core from the environment. The opening of the sensor area in the protective layer is done, after a photolithographic process by wet chemical etching. Several MZI configurations were designed varying the separation between arms and the Y-junction parameters. All the devices are symmetric with two different divisor shapes: in the first case the Y-junction is formed with straight arms and opening angle of $1^{\circ}$. In the second case, the divisor is shaped with circular bends with radii of 5, 20 and 80 $\mathrm{mm}$. In all cases, the separation between the sensor and reference arms is of $100 \mu \mathrm{m}$ to avoid coupling between modes 
travelling through both branches. In one arm, a sensor area with different lengths $(6,10,15$ and $20 \mathrm{~mm})$ is created. The total length of the device is $35 \mathrm{~mm}$. Finally the sensors are cut in individual pieces and polished for light coupling by end-face. The total length of the sensor is $35 \mathrm{~mm}$. The final structure is shown in Fig. 6.

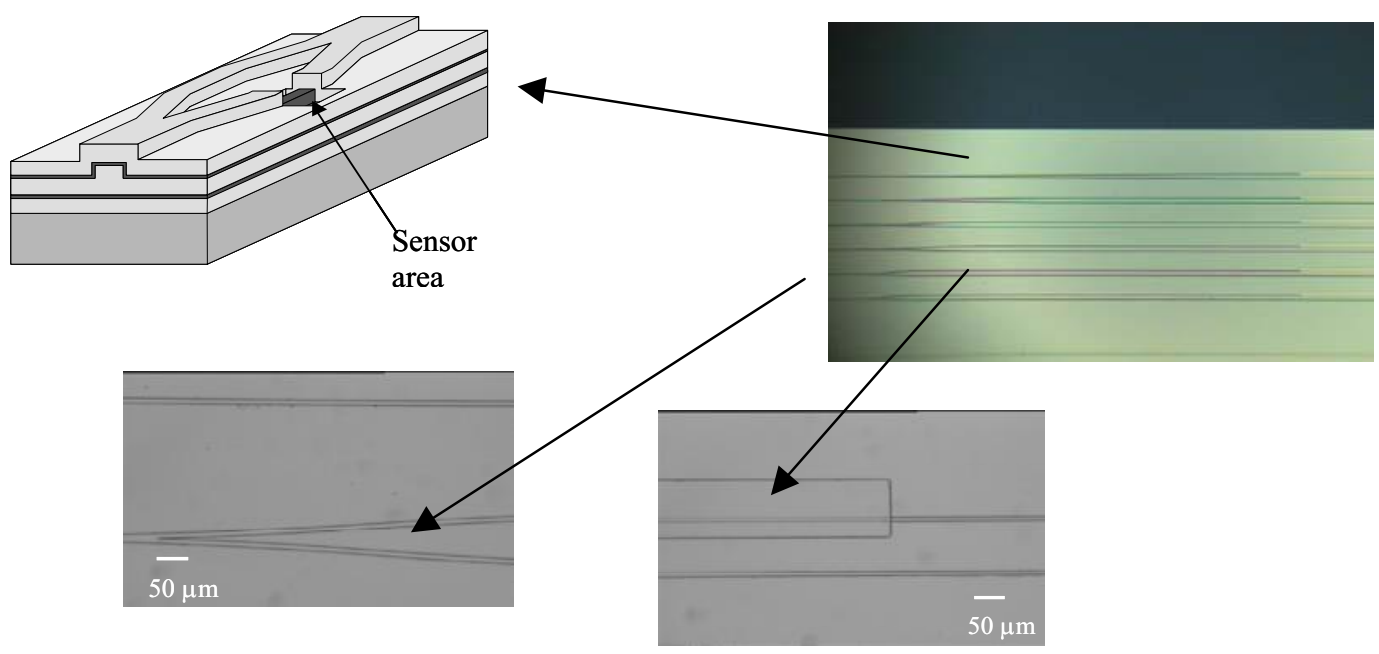

Fig..6 Integrated Mach-Zehnder interferometer: details of the MZI Y-junction and sensor area.

\section{OPTICAL CHARACTERISATION}

The optical characterisation (see Fig 7) is performed with polarized light (TE or TM) from a diode laser $(\lambda=0.670 \mu \mathrm{m})$ that is coupled to a single-mode optical fibre ( $3.8 \mu \mathrm{m}$ core diameter). The end of the mono-mode fibre is placed in front of the waveguide rib face to couple light into the sensor structure (end-fire coupling). Light is collected by a multi-mode standard optical fibre (50 $\mathrm{mm}$ core diameter) connected to a silicon photodiode and data acquisition system. Precise translation stages are used for the accurate alignment of the components.

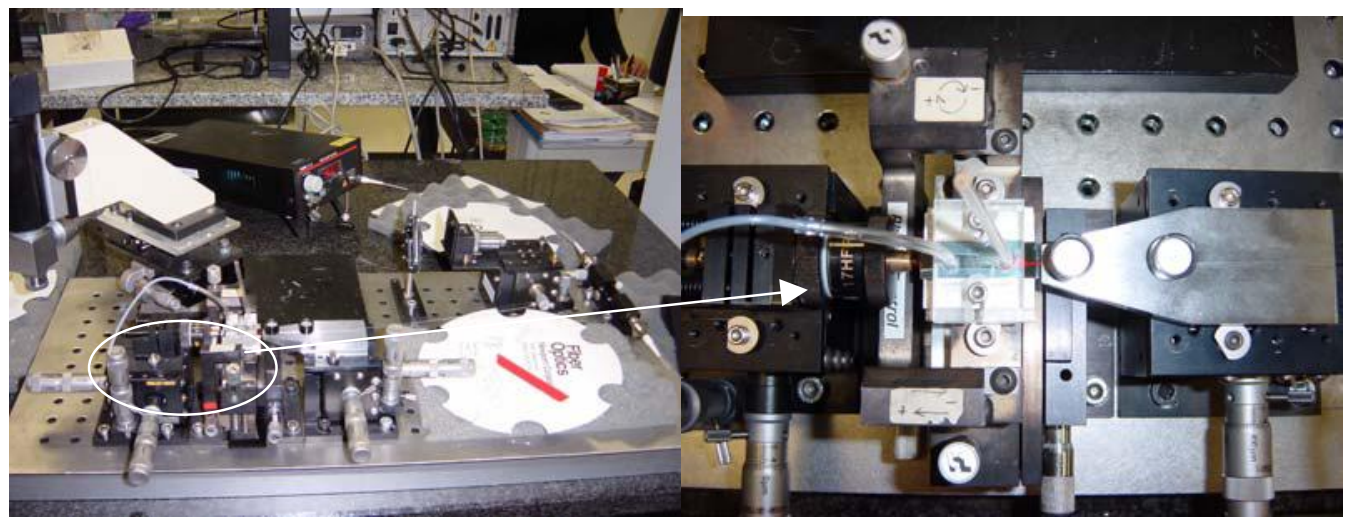

Fig.7. Optical bench for the optical and biochemical characterization of the MZI devices.

An experimental study was made to analyse the guiding conditions of the fabricated structures. Straight waveguides and Y-couplers were used to measure the propagation losses and modal behaviour as a function of the waveguide width and 
the polarization. (a) For TIR waveguides, propagation losses, measured by the Fabry-Perot resonance method, vary between $0.15-0.25 \mathrm{~dB} / \mathrm{cm}$ for TE polarization and between $0.2-0.35 \mathrm{~dB} / \mathrm{cm}$ for TM polarization. Monomodal behaviour is observed for the devices with $4 \mu \mathrm{m}$ of rib width (see Fig. 8). For wider waveguides a second propagating mode could be excited. With these results we can conclude that the optimum interferometers for sensing applications should have a rib width of $4 \mu \mathrm{m}$ to assure monomode behaviour, while low losses are assured with couplers designed with circular arms of $\mathrm{R}=80 \mathrm{~mm}$.

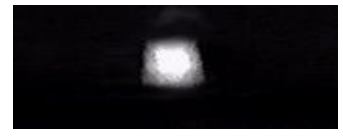

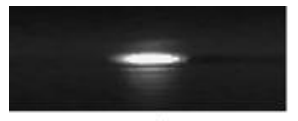

a)

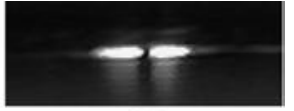

b)

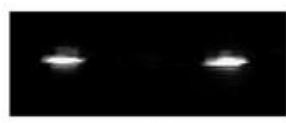

c)

Fig.8. MZI Monomode behaviour (left) ARROW-MZI of a rib width of $4 \mu \mathrm{m}$ (right) TIR-MZI (a) rib width of $4 \mu \mathrm{m}$ (b) rib width of $5 \mu \mathrm{m}$ (c) Y-coupler of width of $4 \mu \mathrm{m}$

(b) For ARROW waveguides single-mode behaviour is observed for rib widths below $8 \mu$ m. Attenuation losses are close to $0.5 \mathrm{~dB} / \mathrm{cm}$ for both polarizations.

\section{CHEMICAL CHARACTERISATION}

Solutions with different refractive indexes are employed for evaluating the sensor sensitivity. Several solutions were prepared with different glucose concentrations in water, with refractive indexes varying from 1.3325 to 1.4004 $(\Delta \mathrm{n}=0.0002)$, as determined by an Abbe refractometer operating at $25^{\circ} \mathrm{C}$. A PMMA mechanized flow cell (with a channel width of $3 \mathrm{~mm}$, a depth of $100 \mu \mathrm{m}$ and a length of $15 \mathrm{~mm}$ ) is clamped onto the interferometer and a flow

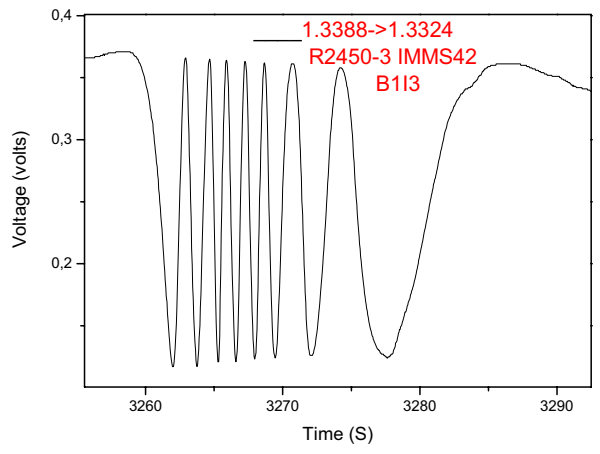

Fig. 7. Interferometric signal from a TIR-MZI device due to a refractive index change

injection system is used to deliver the glucose solutions into the sensor area. The solutions with varying refractive indexes were introduced alternatively, and the change induced in the MZI device can be seen in Fig. 7 for one concentration. With these measurements, a calibrating curve was evaluated where the phase response of the sensor is plotted versus the variation in the refractive index, as is depicted in Fig. 8 for a TIR-MZI device. For the TIR device, the lower detection limit measured was $\Delta n_{o, \min }=7 \cdot 10^{-6}$ that means an effective refractive index of $\Delta N=4 \cdot 10^{-7}$. The detection limit has been estimated measuring the signal-to-noise ratio (around $20 \mathrm{~dB}$ ) and considering the most 
favourable case in which the interferometer is located at its quadrature point. Under this conditions, we estimate that the lowest phase shift measurable would be around $0.01 \cdot 2 \pi$. The detection limit value corresponds to a surface sensitivity around $2 \times 10^{-4} \mathrm{~nm}^{-1}$, close to the maximum surface sensitivity reported up to now.

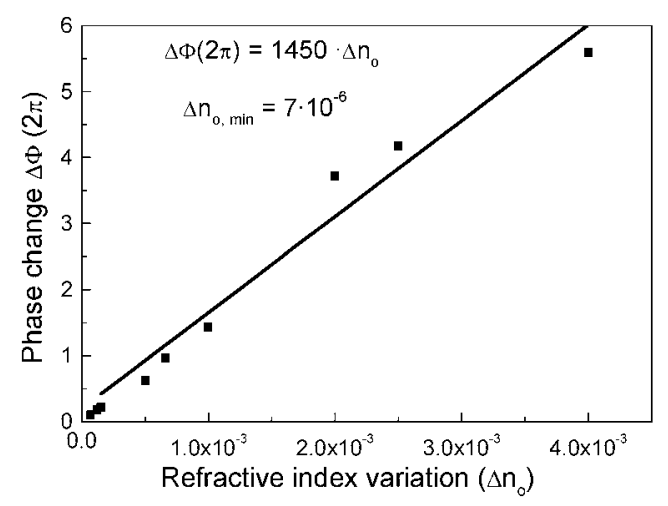

Fig. 8. Sensitivity evaluation of a TIR-MZI nanodevice by using glucose solutions of varying refractive indices.

For the ARROW-MZI devices, for a MZI sensor with $3 \mu \mathrm{m}$ of core thickness and a sensor area length of $15 \mathrm{~mm}$, the maximum sensitivity obtained is $\frac{d \Delta \Phi(2 \pi)}{d n}=480$, which corresponds to a minimum detectable refractive index variation of $\Delta n_{o, \min }=2 \cdot 10^{-5}\left(\Delta N=4 \cdot 10^{-7}\right)$.

\section{BIOSENSING APPLICATIONS}

\subsection{BIOLOGICAL IMMOBILISATION AT NANOMETER SCALE}

For biosensing purposes, a layer of receptor molecules (as proteins or DNA) that are capable of binding the analyte molecules in a selective way, has to be previously immobilised on the biosensor surface. When the complementary analytes are flowing over the surface can be directly recognised by the receptor through a change in the properties of the sensor. In this way, the interacting components do not need to be labelled and complex samples can be analysed without purification.

Fig. 9. Scheme of the immobilization procedure at nanometer scale employed for the attachment of the receptor at the sensor surface.

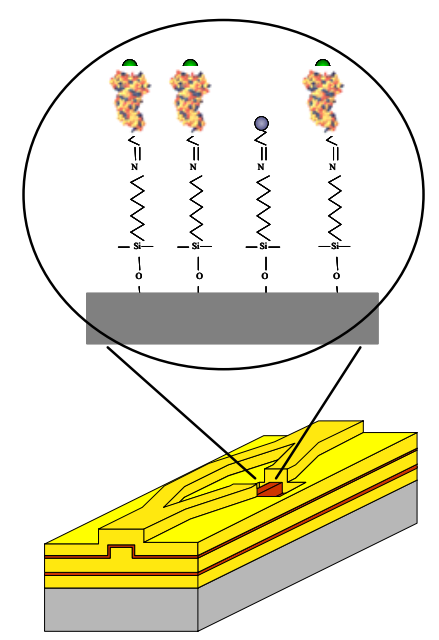


The immobilization of the receptor molecule on the sensor surface is a key point for the final performance of the sensor. The immobilization procedure must be stable and reproducible and must retain the stability and activity of receptor. In this way can allow high sensitivity levels and miniaturization. Generally, direct adsorption is not adequate, giving significant losses in biological activity and random orientation of the receptors. One of the most promising strategies is the covalent immobilization on surfaces. This can be applied for silicon surfaces, using silane monolayers covalently attach to the $\mathrm{SiO}_{2}$ or $\mathrm{Si}_{3} \mathrm{~N}_{4}$ sensor surfaces The reproducibility and regeneration of the sensor surface requires the application of a defined immobilization procedure, and then the sensor surface may be prepared to attach covalently the receptor. Several aspects must be taken into account in the development of the immobilization procedures as the nonspecific interactions, an optimised surface density of the receptor in order to prevent steric hindrance phenomenon or the regeneration of the receptor. We have developed immobilisation procedures at nanometer-scale which try to fulfil most of the requirements described above based on silanization and esterification chemistries. In Fig. 9 a scheme of the specific immobilization procedure for proteins based on silanization can be observed.

\subsection{ENVIRONMENTAL APPLICATIONS}

We have applied the MZI biosensors for the detection of the insecticide carbaryl. Carbaryl is an N-methylcarbamate pesticide extensively used as a broad-spectrum insecticide. The immunoassays developed to monitor this pollutant require the use of three different components. The critical part is the monoclonal antibody (LIB-CNH45 Mab), since it is responsible for the sensitivity and specificity of the analyte to determine. The second component is the analyte (antigen) to detect, which is unable to produce directly an immune response due to its low molecular weight ( 200D). Therefore, it is necessary to design the third compound: the hapten, which can be covalently bound to a large carrier, usually a protein. A hapten is a derivative of the analyte, in this case carbaryl, with similar geometry and structure, which contains an appropriate group $\left(\mathrm{COOH},-\mathrm{NH}_{2}\right)$ for attachment to the carrier protein $(\mathrm{OVA})$. Once the haptencarrier conjugate has been formed, antibodies can be produced by animal immunization. To achieve higher specificity and lower cross-reactivity, monoclonal antibodies, obtained unlimitedly by the hybridoma technique are preferred to heterogeneous polyclonal antibodies.

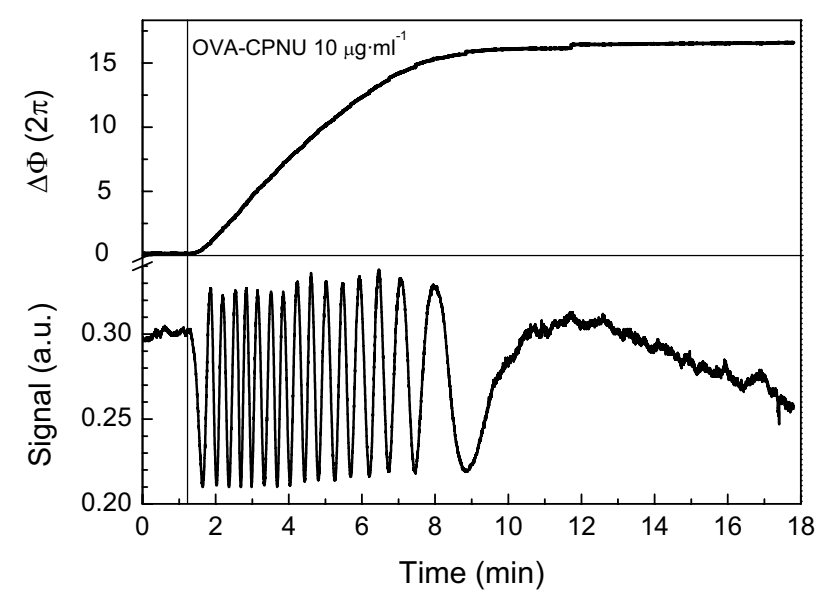

Fig.10. Immobilization of the receptor at nanometer scale by covalent attachment

Carbaryl determination requires the immobilization of the hapten-carrier conjugate (carbaryl OVA-CPNU) and the subsequent addition of the antibody LIB-CNH45. The immobilization procedure used is in this case was an esterification process of the silicon nitride sensor surface. A concentration of $10 \mu \mathrm{g} \cdot \mathrm{ml}^{-1}$ in a buffer solution (PBST, Phosphate Buffered Saline Tween) with $\mathrm{pH} 7$ at a constant flow rate of $20 \mu \mathrm{l} \cdot \mathrm{min}^{-1}$ is introduced. As it is observed in Figure 11 the phase change, in the first part of the process, is fast and as the surface is being progressively occupied, the phase response $\Delta \Phi$ varies more slowly. The total phase change is $16.2 .2 \pi$, that corresponds to the adsorption of a homogeneous antigen monolayer of average thickness $\mathrm{d}_{\ell} \approx 3.2 \mathrm{~nm}$ (surface covering of $1.9 \mathrm{ng} \cdot \mathrm{mm}^{-2}$ ). A concentration of $10 \mu \mathrm{g} \cdot \mathrm{ml}^{-1}$ of the antibody (LIB-CNH45) is flowed through the sensor, giving an additional phase change of $\Delta \Phi=$ 
$5.2 \pi$ due to the immunoreaction as it is shown in Figure 11. After rinsing with PBST, the antibodies that have not reacted are washed out, being the net sensor response of $\Delta \Phi=0.4 .2 \pi$ (surface covering of $0.33 \mathrm{ng} \cdot \mathrm{mm}^{-2}$ ).

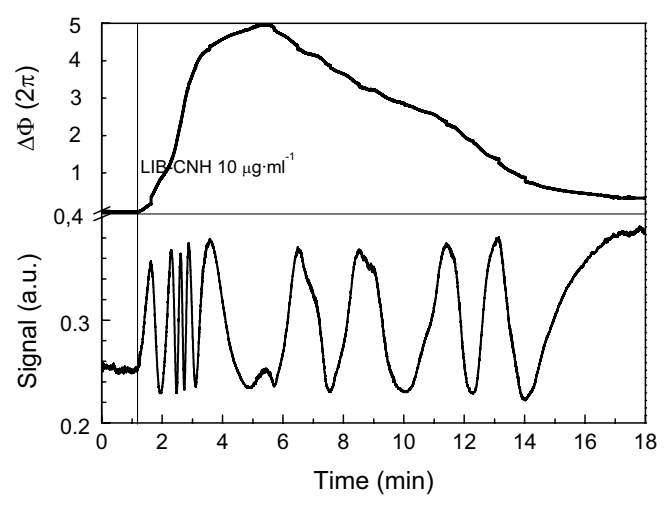

Fig.11. Immunoreaction with the pollutant.

\section{Towards a CMOS compatible lab-on-a-chip microsystem}

For development of a complete microsystem several units must be incorporated on the same platform: (i) the micro/nanodevices, (ii) the flow cells and the flow delivery system, (iii) a modulation or compensation system for translating the interferometric signals in direct ones, (iv) integration of the light sources and the photodetectors (v) CMOS processing electronics. Several steps are undergoing for achieving that. Until now, we have fully developed the first step (i) but steps (ii) and (iii) are under development as well. The microflow cells are specifically designed and fabricated using a novel fabrication method ${ }^{8}$ of 3-D embedded microchannels using the polymer SU-8 as structural material. Several modulation schemes into the reference arm (as magneto-optical compensation) are under study ${ }^{9}$. Integration of sources will be achieved by connection of optical fibers or using embedded gratings.

\subsection{Microfluidic system on SU-8}

3-D microflow cells are specifically fabricated using a novel fabrication method by using a novel low temperature (100 ${ }^{\circ} \mathrm{C}$ ) CMOS compatible wafer bonding process. This fabrication technique has been developed for fabricating complex 3-D embedded microchannel paths. The technology is based on adhesive polymer bonding of two photolithography patterned SU-8 layers. These layers are spin coated and photopatterned onto two separate wafers (i) a Si processed wafer, with the MZI biosensors and (ii) a Pyrex or Silicon wafer. The photopatterned SU-8 layers are brought into contact, pressed together and heated above the glass transition temperature of the SU-8, causing the two polymer layers to become bonded (glued) together. By this fabrication method the microflow sealed cells are fixed on top of the MZI biosensors and will be used in future measurements. A schematic of the process is shown in Figure 12.

A special treatment during the photolithography process of the layers causes the SU-8 polymer film to weld together after defining the 2-D photopatterned structures. High quality, uniformity ( $0.3 \%$ thickness StD across 4 inch wafer) with good adhesive properties SU-8 photopatterned thick films have been obtained. These SU-8 films are suitable for bonding applications.

Once the photopatterned SU-8 layers have been generated by the photolithography processes, the wafers were bonded together to obtain the 3-D sealed microchannels between them. A wafer-level bonding protocol have been established using a commercial bond equipment. Firstly, the wafers were loaded into the bond chamber separated by spacers. Then, the bond chamber was evacuated to $10^{-3}$ mbar. Prior to contact, two heaters (bottom and top) started to heat uniformly the wafers at a temperature of $85^{\circ} \mathrm{C}$ for three minutes, to enable moisture evaporation and prevent any void formation. Afterwards, the wafers were brought into contact and the two heaters start to heat the wafers at a temperature of 100$120^{\circ} \mathrm{C}$, and applying an optimized pressure of $300 \mathrm{KPa}$ for 20 minutes. These bonding process parameters have been chosen in order to achieve a strong and void-free bond. The bond strength was characterized by tensile strength tests. 
The bonding stacks were diced into $500 \times 500 \mathrm{~mm}^{2}$ pieces for tensile strength measurement with a DAGE-4000 shear test tool adaptive for tensile strength measurement. Bond strength up to $8 \mathrm{MPa}$ have been obtained.

1. Bottom wafer. TIR process waveguides

2. SU-8 spin coating $(20-50 \mu \mathrm{m})$

3. SU-8 UV photolithography
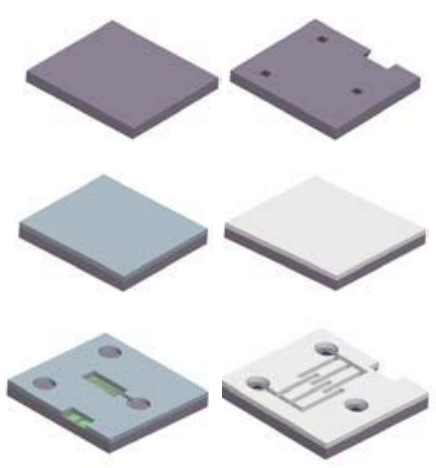

4. Bonding Process

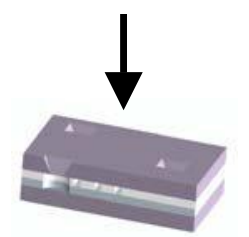

1.Top wafer. Si or Pyrex wafer

2. SU-8 spin coating $(20-50 \mu \mathrm{m})$

3. SU-8 UV photolithography

Fig.12. Scheme of the fabrication process. After spin coating, both wafers were photopatterned. After the 2-D microchannels are defined, the wafers are brought into contact inside the bonding chamber. The bonding process is performed.

Figure 13 (a and b) shows an example of a bonding using a Si processed wafer (300 nm LPCVD Si $\mathrm{N}_{4}$ over $2 \mu \mathrm{m} \mathrm{Si}$ themal oxide) with a Pyrex wafer. The right side figure is magnified 200 times using an optical microscope. The crossing microchannels that connect with the MZI's sensing arms are showed. The use of Pyrex wafers will allow the optical inspection of the flow during the biosensing evaluation.

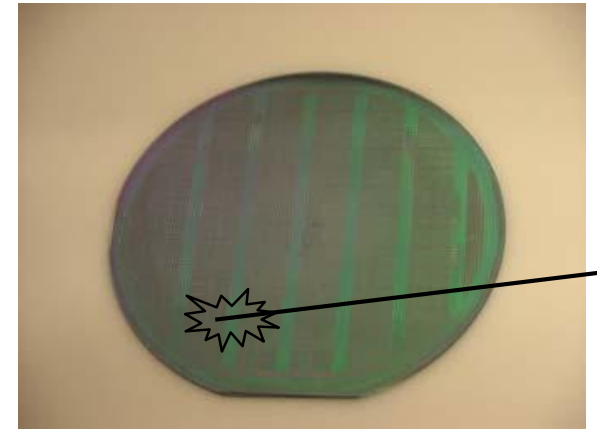

Figure 13 a). A wafer bonding between a Pyrex and a Si processed wafer (LPCVD Si3N4 (300 nm)/ Thermal Si02 (2 $\mu \mathrm{m}) / \mathrm{Si})$.

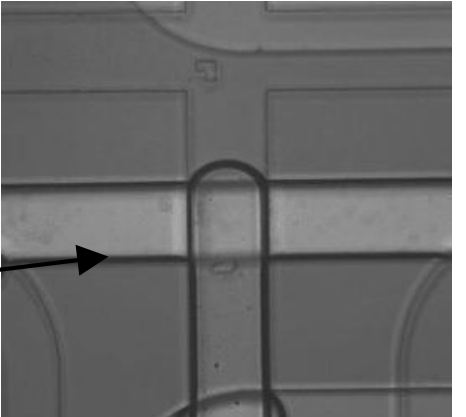

Figure 13 b). Optical photograph through the Pyrex wafer. Crossing Microchannels filled with DI water are showed.

Figures 14-17 show the cross-section of the two level interconnected embedded channels for Si-Si and Si-Pyrex bond. We have achieved perfect vertical smooth walls, with a total intermediate thickness from 40 to $60 \mu \mathrm{m}$ and from 10 to 250 - $\mu \mathrm{m}$-wide. This channel structures are been optimized for biosensing purposes. 


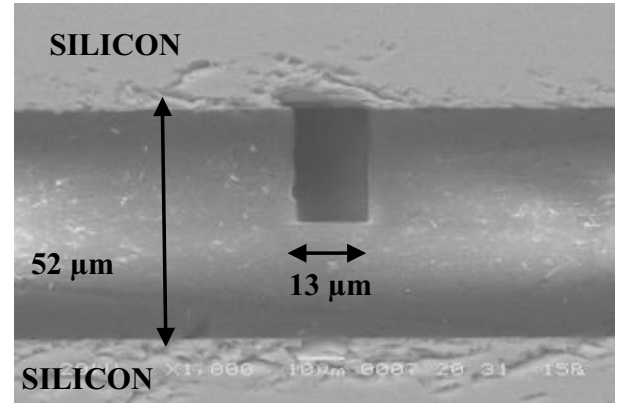

Figure 14. SEM photograph of the bonding interface between $\mathrm{Si}$ wafers. It is showed an embedded microchannel

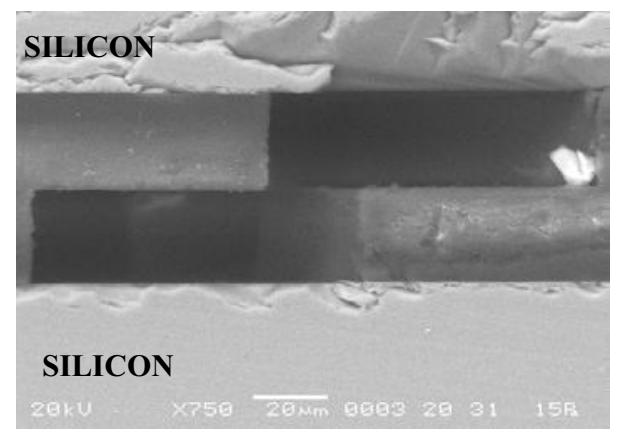

Figure 16. Cross section SEM photograph of 3-D embedded microchannels between Si wafers

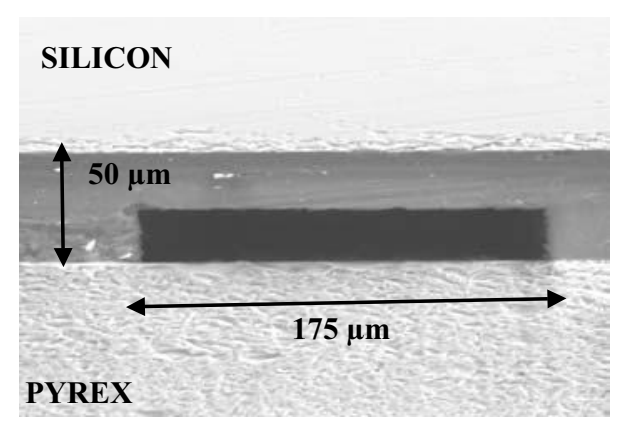

Figure 15. SEM photograph of the bonding interface between Si and Pyrex wafers.

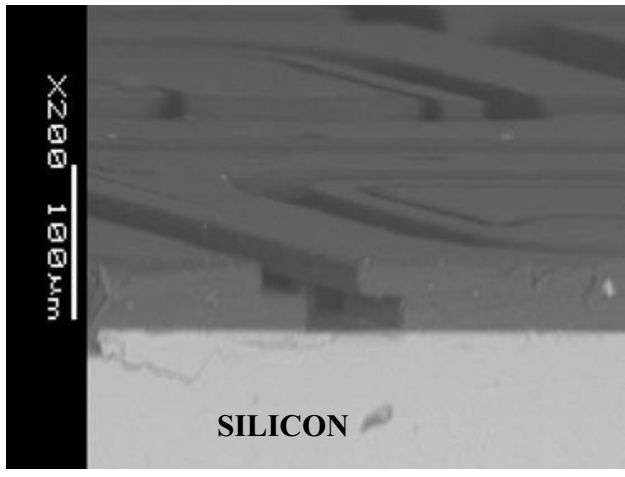

Figure 17. SEM photograph of 3-D network microchannels after release the top wafer

\subsection{Phase compensation based on magneto-optic effects}

Due to the periodicity of the output MZI signal, the detected intensity variation could not be proportional to the measurand and, therefore, it is necessary to develop a signal processing scheme to overcome this disadvantage. The problems with the periodicity of the output interferometer signal can be solved by incorporating a modulation system. Using different operation principles (electro-optic effect, thermo-optic effect, etc.) a phase change can be induced in one of the MZI branches respect to the other by applying an external signal, in such a way that the interferometer can be tuned to the quadrature condition ${ }^{6}$. Our approximation is to develop a compensation system based on magnetooptical effects.

The application of a magnetic field in a magnetic material modifies the propagation properties of the light (magnetooptic effect). The magneto-optic phase compensation is based on the linear magnetic birrefringence induced when a light beam propagates perpendicular to the direction of the magnetization, also called Voigt or Cotton-Moutton effect. The materials that show high magneto-optic effect are the ferromagnetic materials. However, such ferromagnetic materials present a strong absorption and cannot be used in the waveguides. This problem can be overcome by depositing a magnetic layer formed by magnetic nanoparticles (of $\mathrm{Fe}, \mathrm{Co}, \mathrm{Fe}_{2} \mathrm{O}_{3}, \ldots$ ) embedded in a dielectric matrix $\left(\mathrm{SiO}_{2}\right)$ over the core of the waveguide. These systems show high magneto-optic effects maintaining a good transparency.

With this configuration the evanescent field of the light travelling within the waveguide can interact with the magnetic nanoparticles. When a magnetic field is applied perpendicular to the propagation direction of light there is a change of 
the refractive index of the layer. This refractive index variation will modify the effective refractive index of light, giving rise to a phase shift between the branches of the interferometer.

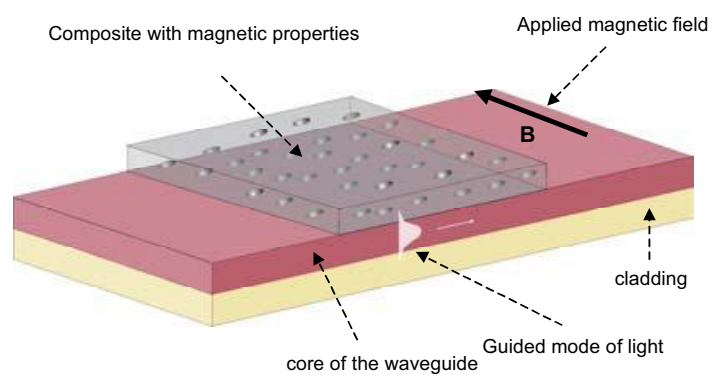

Fig. 18. Scheme of the magnetooptical modulation system to be incorporated on the reference arm of the MZI devices.

\section{CONCLUSIONS}

We have developed optical micro/nanobiosensors based on Silicon microelectronics technology. The devices make use of an integrated MZI configuration fabricated with TIR and ARROW waveguides. The main features of these waveguides must be monomode behaviour and high surface sensitivity and for achieving that the parameters of the waveguides must be carefully chosen. We have presented experimental results of two different MZI biosensors:TIR and ARROW. For the MZI-TIR sensor the lower refractive index detection limit $\Delta n_{o, \min }=7.10^{-6}$. For the MZI-ARROW sensor the lower refractive index detection limit $\Delta n_{o, \min }=2.10^{-5}$. Both devices have been used for the detection of environmental pollutants. For a further integration in a complete microsystem, several steps are underway, as the integration with a novel microfluidics system based on SU-8 technology and the development of a new modulation scheme based on magneto-optical effects.

\section{ACKNOWLEDGMENTS}

This work has been supported by the national project BIO2000-0351-P4. B Sepúlveda acknowledges CSIC-I3P program the financial support for Ph. D. studies. The authors want to thank to Dr. A. Montoya (LIB-UPV) for the immunoreagents.

\section{REFERENCES}

1. C. Domínguez, L.M. Lechuga and J.A. Rodríguez. Integrated optical chemo- and Biosensors. In Integrated Analytical Systems. Ed. S. Alegret. (2003). Elsevier Science BV. Amsterdam (The Netherlands). ISBN: 0-44451037-0

2. Kooyman, R.P.H. \& Lechuga, L.M., "Immunosensors based on Total Internal Reflectance", Ch. 8, Handbook of Biosensors: Medicine, Food and the Environment, ed. E. Kress-Rogers, CRC Press, Florida (USA), pp. 169196, 1997.

3. J. Homola, S.S. Yee and G. Gauglitz. "Surface plasmon resonance sensors: review”. Sens. Act. B, 54 (1999) 315.

4. M. Wiki, H. Gao, M. Juvet and R.E. Kunz. "Compact integrated optical sensor system”. Biosens. Bioelec. 16 (2001) 37-45

5. T. Kinning and P. Edwards. "The resonant mirror optical biosensor", Ch. 8 In Optical Biosensors: present and future. Ed. F.S. Ligler and C.A. Rowe Taitt. Elsevier, NL (2002). 
6. L. M. Lechuga, F. Prieto and B. Sepúlveda. "Interferometric Biosensors for environmental pollution detection". In Optical Sensors for industrial and environmental applications. Ed. R. Narayanaswamy and O.S. Wolfbeis. 2003. Springer (Springer Series on Chemical Sensors and Biosensors). ISBN: 3-540-40886-X

7. L.M. Lechuga, B. Sepúlveda, A. Llobera, A. Calle and C. Domínguez. "Integrated optical silicon IC compatible nanodevices for biosensing applications". Proceedings of SPIE (The International Society for Optical engineering). Microtechnologies for the New Millennium 2003: Bioengineered and Bioinspired Systems 5119 (2003)140-148.

8. F.J. Blanco et al. "A Novel low temperature CMOS compatible Full wafer bonding process for the fabrication of 3D embedded microchanels using SU-8 Photoresist" Proceed. SPIE 2003 Microelectronics, MEMS and Nanotechnology International Conference. 12-14 December 2003 (Perth, Australia) (In press)

9. B. Sepúlveda and L. M. Lechuga. "Matrix Analysis of Discontinuities in Non-Reciprocal Waveguides: Analytical Description for Magneto-Optical Slab Waveguides. J. Lightwave Tech. (Submitted)

10. K. Tiefenthaler, W. Lukosz, "Sensitivity of grating couplers as integrated-optical chemical sensors," J. Opt. Soc. Am. B, vol. 6, pp. 209-220, 1989.

11. F. Prieto, A. Llobera, A. Calle, C Domínguez and L.M. Lechuga. "Design and analysis of Silicon Antiresonant Reflective Optical Waveguides for Highly Sensitive Sensors". J. Lightwave Tech. 18 (7), 966-972 (2000).

12. F. Prieto, L.M. Lechuga, A. Llobera, A. Calle and C Domínguez. "Optimised Silicon Antiresonant Reflecting Optical Waveguides for Sensing Applications". J.of Lightwave Tech. 19 (1), 75-83 (2001).

13. F. Prieto, B. Sepúlveda, A. Calle, A. Llobera, C. Domínguez and L.M. Lechuga. "Integrated Mach-Zehnder Interferometer based on ARROW structures for biosensor applications". Sensors and Actuators B 92 (2003) 151-158. 